Bioscientia Medicina: Journal of Biomedicine \& Translational Research

Journal Homepage: www.bioscmed.com

\title{
Iron-Deficiency Anemia Related to Ancylostoma duodenale Infection in 67 Years Old Female Patient
}

\section{Widodo Adi Prasetyo ${ }^{1 *}$, Armen Ahmad ${ }^{1}$}

${ }^{1}$ Department of Internal Medicine, Faculty of Medicine, Universitas Andalas/ Dr. M. Djamil General Hospital, Padang, Indonesia

\author{
A R T I C L E I N F O \\ Keywords: \\ Ancylostomiasis \\ Anemia \\ Iron deficiency \\ Ancylostoma duodenale \\ Ferrous sulfate \\ *Corresponding author: \\ Widodo Adi Prasetyo \\ E-mail address: \\ widodoadiprasetyo@gmail.com
}

All authors have reviewed and approved the final version of the manuscript.

https://doi.org/10.37275/bsm.v6i3.459

\begin{abstract}
A B S T R A C T
Background. Ancylostomiasis is an infection caused by hookworms. It is estimated that hookworms infect 1.2 billion people worldwide and cause higher morbidity than other types, mainly due to iron deficiency anemia. Hookworm infection increases with age. The main clinical manifestations are abdominal pain, features of iron deficiency anemia and hypoproteinemia. Diagnosis is based on the finding of characteristic eggs in the stool.

Case presentation. A 67-year-old female patient had been administered into the Internal Medicine Ward of Dr. M. Djamil Padang Hospital with the main complaint of weakness, fatigue, and lethargy which had increased since 3 days before being admitted to the hospital. The patient also had complaints of pale skin, fever, heartburn, decreased appetite and weight loss. The patient was admitted to the hospital with awareness of compost mentis, cooperative, and his general condition appeared to be moderately ill, blood pressure 130/90 $\mathrm{mmHg}$, pulse rate $104 \mathrm{x} /$ minute, regular pulse, adequate filling, respiratory rate 22 $\mathrm{x} /$ minute, temperature $37.8 \mathrm{C}$. On physical examination, the conjunctiva of the pantient's eyes were anemic. Laboratory examination revealed Hemoglobin 4 $\mathrm{g} / \mathrm{dl}$, Hematocrit 16\%, MCV/MCH/MCHC 62fl/15pg/25g/dL, Reticulocytes $0.5 \%$, SI/TIBC $22 / 315 \mathrm{mg} / \mathrm{dL}$, Ferritin $8.5 \mathrm{ng} / \mathrm{mL}$, and peripheral blood smear showing severe hypochromic microcytic anemia and relative eosinofilia. Ancylostoma duodenale worm eggs were found on routine stool examination. Final diagnosis was Severe microcytic hypochromic anemia caused by an iron deficiency and Ancylostomiasis. Severe microcytic hypochromic anemia caused by iron deficiency and ancylostomiasis was established based on the patient's history, physical examination and laboratory investigations.

Conclusion. Hookworms infect many people worldwide and cause high morbidity, mainly due to iron deficiency anemia. The highest prevalence is found in the tropics and subtropics, including Indonesia. The main clinical manifestations are abdominal pain, features of iron deficiency anemia and hypoproteinemia. Diagnosis is based on the finding of characteristic eggs in the stool. Albendazole $400 \mathrm{mg}$ single dose resulted in $80 \%$ cure and a dose of 200 $\mathrm{mg}$ /day for 3 days gave $100 \%$ cure. Treatment of anemia is by administering ferrous sulfate or ferrous gluconate orally $200 \mathrm{mg}$ three times a day and continued for up to 3 months after normal hemoglobin levels are achieved to maintain iron stores.
\end{abstract}

\section{Introduction}

Ancylostomiasis is caused by 2 types of hookworms, namely Ancylostoma duodenale and Necator americanus. ${ }^{1}$ It is estimated that hookworms infect 1.2 billion people worldwide and cause higher morbidity than other types of worms, mainly due to iron deficiency anemia. Hookworm infection increases with age. The highest prevalence is found in the tropics and subtropics of Sub-Saharan Africa, South China, the Pacific and Southeast Asia. A. duodenale is native to Southern Europe, North Africa, Northern India, 
Northern China and Japan. Due to population migration, this worm is also found in coastal areas of Peru, Chile, Paraguay, Western Australia, South India, Malaysia, Indonesia, Philippines, Polynesia, Micronesia, parts of West Africa. ${ }^{1-3}$

Ancylostoma duodenale measures 0.7 - $1.1 \times 0.4$ $0.5 \mathrm{~cm}$ (male) and $1-1.3$ x $0.6 \mathrm{~cm}$ (female), cylindrical, white, gray or reddish brown in color. Female worms produce 25,000 - 35,000 eggs per day. Adult worms live in the human small intestine for 1-3 years. The eggs are excreted in the feces, hatch into rhabditiform larvae in moist soil, develop into filariform larvae. In moist soil the larvae can survive for 2 years. These worms infect humans through the penetration of larvae through the skin. Stage 3 larvae penetrate the blood vessels, reach the lungs on the third day, penetrate the alveoli into the bronchioles, go up the trachea into the esophagus, then through the stomach and reach the small intestine on the seventh day. Within 3 weeks, the worms become adults and the female worms begin to produce eggs. $A$. duodenale can infect humans through ingestion of worm larvae in addition to penetrating the skin. A. duodenale is able to survive in the form of larvae in the body of the host for months before developing into adult worms so as to bridge the mucin where conditions do not allow for transmission. In general, transmission occurs percutaneously or by ingestion of contaminated food. A small number of cases occur due to eating raw meat containing $A$. duodenale larvae. The incubation period is $40-100$ days. ${ }^{2,3}$

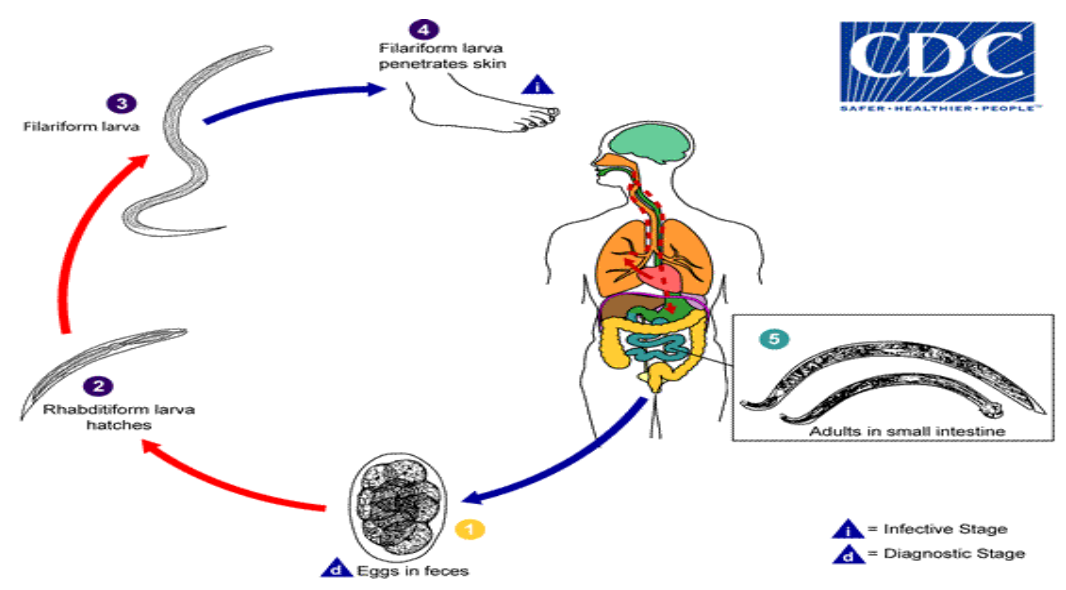

Figure 1. Life cycle of Ancylostoma duodenale

Hookworm infection in humans is characterized by eosinophilia and elevated IgE. One study also showed an increase in IgG, IgA, and IgM, production of Th2 and Th1 cytokines. Immunity to this worm infection is partially protective. Manifestations according to the life cycle of worms from larvae penetrate the skin to become adult worms. At the entry site of the larvae, there is a vesicular rash and itching. After 2 weeks pulmonary symptoms appear, namely dry cough, asthma, fever, hypereosinophilia. The main clinical manifestations are abdominal pain, features of iron deficiency anemia and hypoproteinemia. The attachment of worms to the mucosa and submucosa of the small intestine causes rupture of the intestinal capillaries and arterioles. In addition, these worms suck blood actively with a frequency of 120-200 times per minute and produce inhibitors of factor $\mathrm{Xa}$, factor VIIa, anti-platelet substances. A. duodenale causes blood loss estimated at $0.15 \mathrm{cc}$ per day. In severe infections, 500-1000 worms can be found in the intestines. Patients can experience very low hemoglobin up to $2 \mathrm{~g} / \mathrm{dL}$. $^{4-7}$

Diagnosis is based on the finding of characteristic eggs in the stool. With the Kato-Katz or McMaster method, the severity of infection can be estimated by counting the number of eggs per gram of feces in samples taken over several days. Severe infection is determined if 4000 eggs / gram is obtained in fecal samples. ${ }^{8}$

Treatment is aimed at eliminating parasites and 
treating anemia. Albendazole $400 \mathrm{mg}$ single dose resulted in $80 \%$ cure and a dose of $200 \mathrm{mg} /$ day for 3 days gave $100 \%$ cure. Alternatives are mebendazole $500 \mathrm{mg}$ single dose, pyrantel pamoate $10 \mathrm{mg} / \mathrm{kg}$ for 3 days. Anemia is treated by administering ferrous sulfate or ferrous gluconate orally $200 \mathrm{mg}$ three times a day and continued for up to 3 months after normal hemoglobin levels are achieved to maintain iron stores. In most cases, $\mathrm{Hb}$ levels increase by 1 gram/week. It is also necessary to give folic acid $5 \mathrm{mg} /$ day for 1 month. Another option is parenteral iron (iron dextran or iron poly sorbitol gluconic acid) in patients who cannot tolerate oral iron. ${ }^{9}$

\section{Case Presentation}

A 67 years old female patient is being treated at the Internal Medicine Ward of Dr. M. Djamil hospital in Padang with the main complaint of weakness, fatigue, and lethargy which had increased since 3 days before being admitted to the hospital. The patient also complained of throbbing heartburn that was felt intermittently. There were complaints of intermittent fever and canker sores since 1 week ago. Complaints of weakness, tiredness and lethargy as well as decreased appetite had initially been felt since 2 weeks ago accompanied by watery bowel movements and pale skin. The patient has lost weight, but did not know how much weight has been lost. There were no complaints of nausea, vomiting, chest pain, or urination.

The patient was admitted to the hospital with awareness of compost mentis and was cooperative. Her general condition appeared to be moderately ill, with blood pressure measuring 130/90 mmHg, pulse rate $104 \mathrm{x} /$ minute, regular pulse, adequate filling, respiratory rate $22 \mathrm{x} /$ minute, temperature 37.8 0C. On physical examination, there was atrophy of the papillae of the tongue and anemic conjunctiva. Examination of the lungs, heart, abdomen, back, and limbs were found to be within normal limits.

Laboratory examination revealed Hemoglobin 4 g/dl, Hematocrit 16\%, MCV/MCH/MCHC $62 \mathrm{fl} / 15 \mathrm{pg} / 25 \mathrm{~g} / \mathrm{dL}, \quad$ Reticulocytes $0.5 \%$, SI/TIBC 22/315 mg/dL, Ferritin $8.5 \mathrm{ng} / \mathrm{mL}$, and peripheral blood smear showing severe hypochromic microcytic anemia and relative eosinofilia. On routine stool examination, the stool sample was found to be brown in color, liquid in consistency, and eggs of Ancylostoma duodenale worms were present. Urinalysis examination was found to be within normal limits.

\section{Discussion}

A female patient, 67 years old, has been treated in the Internal Medicine Ward of the Dr. M. Djamil Padang hospital with a final diagnosis of severe microcytic hypochromic anemia due to iron deficiency and ancylostomiasis.

Severe hypochromic microcytic anemia caused by an iron deficiency and ancylostomiasis in this patient was established based on patient history, physical examination and further laboratory investigations. During the patient interview, the patient complains of weakness, lethargy, looks pale, decreased appetite, canker sores. The patient has a history of stepping on the ground barefoot. On physical examination there was atrophy of the papillae of the tongue along with anemic conjunctiva. On routine blood examination, there was a lack of hemoglobin, eosinophilia, low Mean corpuscular volume (MCV), while reticulocytes were within normal limits. Her peripheral blood smear showed a hypochromic microcytic appearance with relative eosinophilia. On examination, serum iron status was low, total iron binding capacity (TIBC) and ferritin levels were within normal limits. On routine stool examination, Ancylostoma duodenale eggs were found. According to Brooker and Hotez, Ancylostomiasis can cause higher morbidity than other types of worms, mainly due to iron deficiency anemia. ${ }^{3,4}$ 


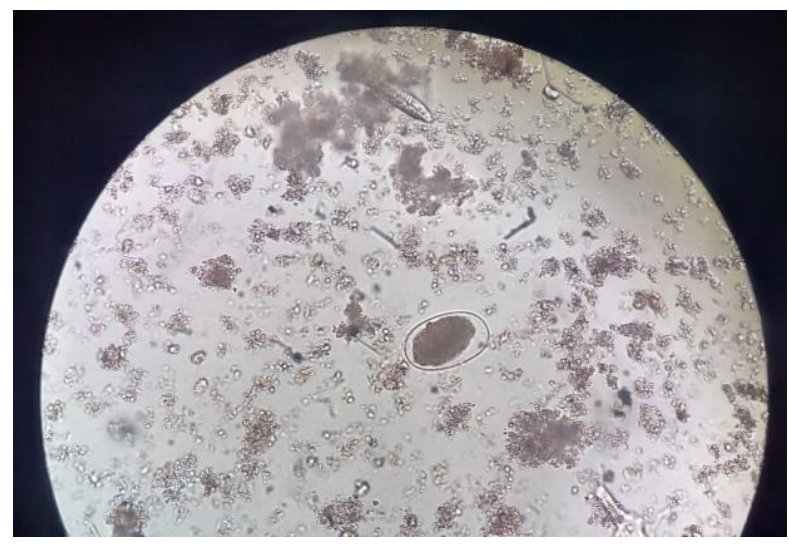

Figure 2. Ancylostoma duodenale eggs

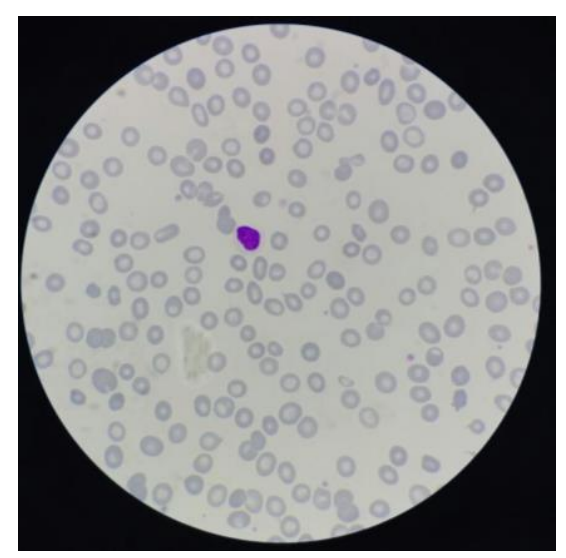

Figure 3. Hypochromic microcytic peripheral blood picture
According to De Silva, Ancylostomiasis increases with age. ${ }^{6}$ This corresponds to this patient who was 67 years old. The environment around the patient's house is dirt and the patient often cleans the area around her house without using footwear. This is one way of the entry of Ancylostoma duodenale worm larvae into the human body, which is through the skin of the foot. However, Ancylostoma duodenale can infect humans through ingestion of worm larvae. Treatment is aimed at eliminating parasites and treating anemia. Albendazole $400 \mathrm{mg}$ single dose resulted in $80 \%$ cure and a dose of $200 \mathrm{mg} /$ day for 3 days gave 100\% cure. Treatment of anemia is by administering ferrous sulfate or ferrous gluconate orally $200 \mathrm{mg}$ three times a day and continued for up to 3 months after normal hemoglobin levels are achieved to maintain iron stores. It is also necessary to give folic acid $5 \mathrm{mg} /$ day for 1 month. 9

The patient was given oxygen therapy $5 \mathrm{~L} /$ minute, Paracetamol 3 x $500 \mathrm{mg}$, Amlodipine 1 x 5 mg, Albendazole 1 x $400 \mathrm{mg}$, Sulfas ferosus 3 × $200 \mathrm{mg}$, Folic acid 1 x $5 \mathrm{mg}$. The patient was given a transfusion of 4 units of PRC. After hospitalization for 4 days, the patient's complaints decreased and her condition improved.

For prevention, a clean lifestyle must be established such as drinking clean water, always cleaning and cooking food thoroughly, washing hands before eating, and always wearing footwear when leaving the house. Antihelminthes can be given to high-risk populations, such as children, pregnant women, lactating women and the elderly. 6,10

\section{Conclusion}

Hookworms infect many people worldwide and cause high morbidity, mainly due to iron deficiency anemia. The highest prevalence is found in the tropics and subtropics, including Indonesia. The main clinical manifestations are abdominal pain, features of iron deficiency anemia and hypoproteinemia. The attachment of worms to the mucosa and submucosa of the small intestine causes rupture of the intestinal capillaries and arterioles. In addition, hookworms suck blood actively with a frequency of 120-200 times per minute, so that the blood loss is estimated at 0.15 cc/day per A. duodenale. Therefore, rapid diagnosis is needed and can reduce morbidity. Diagnosis is based on the finding of characteristic eggs in the stool. Optimal management is also needed to treat the infection and the anemia it causes. Albendazole $400 \mathrm{mg}$ single dose produces $80 \%$ cure and a dose of 200 $\mathrm{mg} /$ day for 3 days gives $100 \%$ cure. Treatment of anemia is by administering ferrous sulfate or ferrous gluconate orally $200 \mathrm{mg}$ three times a day and continued for up to 3 months after normal hemoglobin levels are achieved to maintain iron stores.

\section{References}

1. Stracke K, Jex AR, Traub RJ. Zoonotic ancylostomiasis: An update of a continually 
neglected zoonosis. Am J Trop Med Hygien. 2020; 103(1): 64-8.

2. Suryantari SAA. Prevalence, intensity and risk factors of soil transmittes helminths infections among elementary school students in Ngis village, Karangasem district, Bali. Indo $\mathrm{J}$ Trop Infect Dis. 2019; 7(6): 125-30.

3. Amalia AN, Prasetya H. Study of soil-transmitted helminthiasis prevalence in school children in Surabaya. J Vocation Health Stud. 2017: 1(1): 10-5.

4. Brooker S, Bethony J, Hotez PJ. Human hookworm infection in the $21^{\text {st }}$ century. Adv Parasitol 2004; 58: 197-288

5. Hotez PJ, Brooker S, Bethony JM, Botazzzi ME, Loukas A, et al. Hookworm infection. N Engl J Med 2004; 351: 799-807

6. De Silva NR, Brooker S, Hotez PJ, Montresor A, Engels D, Savioli L. Soil transmitted helminthes infection: updating the global picture. Trends Parasitol 2003; 19: 547-55

7. Albonico M, Montresor A, Crompton DW, Savioli L. Intervention for the control of soil-transmitted helminthiasis in the community. Adv Paraasitol 2006; 61: 31 .

8. Sari MP, Nathasaria T, Majawati ES, Pangaribuan HU. Soil-transmitted helminth infections, anemia and undernutrition among school children in an elementary school in North Jakarta, Indonesia. Majalah Kedokteran Bandung.2020; 52(4): 2661-70.

9. Budiapsari PI, Swastika IK, Masyeni S. Prevalence of soil-transmitted helminths infection in students of Klungkung, Bali, after mass treatment with albendazole. Open Access Maced J Med Sci. 2021; 9: 433-9.

10.Vlaminck J, Cools P, Albonico M, Ame S, Ayana M, et al. Therapeutic efficacy of albendazole against soil-transmitted helminthiasis in children measured by five diagnostic methods.PLoS Negl Trop Dis. 2019; 13(8): e0007471. 